\title{
EXPERIMENTAL MODEL OF HEPATIC STEATOSIS BY FRUCTOSE IN ADULT ZEBRAFISH: A PILOT STUDY
}

\author{
Jéssica Tonin Ferrari ${ }^{1,2}$, Raquel Ayres ${ }^{1,3}$, Thais Ortiz Hammes ${ }^{4}$, \\ Themis Reverbel da Silveira ${ }^{1}$, Carolina Uribe-Cruz ${ }^{1,2}$
}

\section{ABSTRACT}

Introduction: The consumption of fructose has been questioned, since its increase has led to an associated increase in steatosis caused by nonalcoholic fatty liver disease. Despite the advantages presented by the zebrafish as an animal model, at present there are no models of steatosis by fructose in adult zebrafish. The aim of this study is to establish a model of hepatic steatosis by fructose in adult zebrafish.

Methods: Firstly, adult zebrafish were daily exposed to $4 \%$ or $6 \%$ fructose. Then, animals were exposed to $6 \%$ fructose every 2 days. The hepatic lipid accumulation was analyzed by Nile Red and Oil Red O staining.

Results: The daily exposure to $6 \%$ fructose showed increased accumulation of hepatic lipids when compared to $4 \%$ and control groups, but the same concentration showed no difference when the exposure happened every 2 days.

Conclusion: We can suggest the daily exposure to a concentration of $6 \%$ fructose can be considered as a new experimental model of adult zebrafish.

Keywords: Fatty liver; fructose; zebrafish

Fructose consumption has been related to the development and progression of nonalcoholic fatty liver disease (NAFLD) ${ }^{1,2}$. This encompasses a broad spectrum of liver diseases in individuals who do not consume alcohol in amounts considered dangerous to the liver ${ }^{3,4}$. Injuries in NAFLD usually start with simple steatosis and can progress to steatohepatitis, cirrhosis, and eventually hepatocellular carcinoma ${ }^{3}$. In a Brazilian study, the estimated prevalence of hepatic steatosis was $20 \%$ in the general population ${ }^{5}$.

Fructose is found naturally in some foods, such as honey and fruits, and artificially in others, such as corn syrup used in soft drinks and processed food $^{6}$. Studies in animal models with a high fructose diet have shown that it promotes de novo lipogenesis, formation of reactive oxygen species, increased intestinal microbiota, and increased risk of cardiovascular diseases ${ }^{7}$.

Animal models of NAFLD provide important information, not only elucidating the pathogenesis and mechanisms of disease, but also examining the effects of various therapeutic agents ${ }^{8}$. In this aspect, zebrafish (Danio rerio) is an excellent study model due to its small size, easy maintenance, low cost, high proliferation, and because it presents $70 \%$ homology with the mammalian genome $^{9,10}$. Despite these advantages, and considering the relevance of fructose in the diet, at present there is no hepatic steatosis model for fructose in adult zebrafish.

In this study, we evaluated the effect of fructose at different concentrations and exposure times to induce hepatic steatosis.
Clin Biomed Res. 2018;38(2):151-154

1 Laboratório Experimental de Hepatologia e Gastroenterologia, Hospital de Clínicas de Porto Alegre (HCPA). Porto Alegre, RS, Brasil.

2 Programa de Pós-graduação em Gastroenterologia e Hepatologia, Universidade Federal do Rio Grande do Sul (UFRGS). Porto Alegre, RS, Brasil.

3 Programa de Pós-graduação em Saúde da Criança e do Adolescente, Universidade Federal do Rio Grande do Sul (UFRGS). Porto Alegre, RS, Brasil.

4 Serviço de Nutrição e Dietética, Hospital de Clínicas de Porto Alegre (HCPA). Porto Alegre, RS, Brasil.

Corresponding author: Jéssica Tonin Ferrari je_t_f@yahoo.com.br Laboratório Experimental de Hepatologia e Gastroenterologia, Hospital de Clínicas de Porto Alegre (HCPA)

Ramiro Barcelos, 2350. 90035-903, Porto Alegre, RS, Brasil. 


\section{METHODS}

\section{Animals}

Eighty adult wild-type zebrafish of both sexes obtained from an aquarium shop were used. The animals were kept in aquariums, five fish/L, at $28{ }^{\circ} \mathrm{C} \pm 2{ }^{\circ} \mathrm{C}$ with light/dark cycles of $10 / 14$ hours under constant aeration and were fed frozen artemia (BioArtémia, Brazil) in an amount of $5 \%$ of body weight of fish. All the aquariums of the experiment were maintained with fungizone, kanamycin (Gibco, USA), and ampicillin (Blaú, Brazil) to prevent water contamination.

All procedures were performed according to the Brazilian legislation (Law 11.794) and Resolution no. 04/97 of the Research Ethics Committee of Hospital de Clínicas de Porto Alegre, which also concerns animal research (GPPG-HCPA protocol 13-0329).

\section{Experimental Design}

Firstly, 48 animals were divided into three groups ( $n=16 /$ group): groups FR4 and FR6, with $40 \mathrm{~g}$ and $60 \mathrm{~g}$ of fructose/L of water respectively, and CTRL, without addition of fructose in the water. For exposure to fructose, the animals were transferred daily for 2 hours to aquariums with FR4 or FR6 and then returned to aquariums with normal conditions. As a control, a group of animals received the same treatment as the animals of the fructose group. After 14 days, the animals were anesthetized with tricaine (400 mg/L, Sigma Aldrich, USA) and euthanized by exsanguination ${ }^{11}$. Livers were removed and frozen at $-20^{\circ} \mathrm{C}$ for further analysis.

After determining the adequate concentration of fructose to induce hepatic steatosis, the remaining 32 animals were divided into two groups $(n=16)$ : FR6 and CTRL. Exposure to fructose was performed every 2 days, as described above. After 20 days, the animals were anesthetized and euthanized by exsanguination ${ }^{11}$. Livers were removed and frozen at $-20{ }^{\circ} \mathrm{C}$ for further analysis.

\section{Analysis of Liver Lipid Deposits}

For quantification of lipid deposits, pools of three livers were homogenized with PBS ( $20 \mathrm{mg}$ of tissue $/ \mathrm{mL}$ ) (Laborclin, Brazil) and then incubated with Nile Red $\left(1 \mathrm{mg} / \mathrm{mL}\right.$ ) (Sigma, USA) for 15 minutes at $37^{\circ} \mathrm{C}$. The fluorescence of the supernatant was measured in a spectrophotometer (SpectraMax M5 Molecular Devices, USA) at $488 \mathrm{~nm}$ of excitation and $550 \mathrm{~nm}$ of emission ${ }^{12}$.

To evaluate hepatic lipid deposits, the livers were sectioned in a cryostat, mounted on slides, and stained with Oil Red O (Sigma, USA). Microphotographs were obtained through an optical microscope (Olympus, Japan).

\section{Statistical Analysis}

Statistical analyzes were performed using the Kruskal Wallis and the Mann Whitney test and the statistical packages SPSS v. 18 and GraphPhad Prism $v$ 5.0. The significance level was set at $p<0.05$.

\section{RESULTS}

When animals were daily exposed to different concentrations of fructose, we observed that the FR6 group had an increased accumulation of lipids $(0.319 \pm 0.148)$ when compared to the CTRL group $(0.112 \pm 0.283)(p=0.042)$. The FR4 group $(0.184 \pm 0.867)$ did not show a significant difference when compared to the CTRL group (Figure $1 \mathrm{~A}$ ). Oil Red $\mathrm{O}$ staining was positive in the FR6 group but negative in the CTRL group (Figure 1B-C). As shown in the figures, the hepatic tissue sample of group F6 presented accumulations of red-stained lipid droplets, whereas the control group did not.

After these results, the concentration of $6 \%$ fructose was chosen to be evaluated in exposures every 2 days. We observed there was no significant statistical difference between the FR6 and CTRL groups when the animals were exposed to fructose every 2 days $(0.059 \pm 0.177$ vs. $0.030 \pm 0.0353)$ (Figure $2 A$ ). No difference was found between the groups in Oil Red $O$ staining results (Figure $2 B-C$ ).

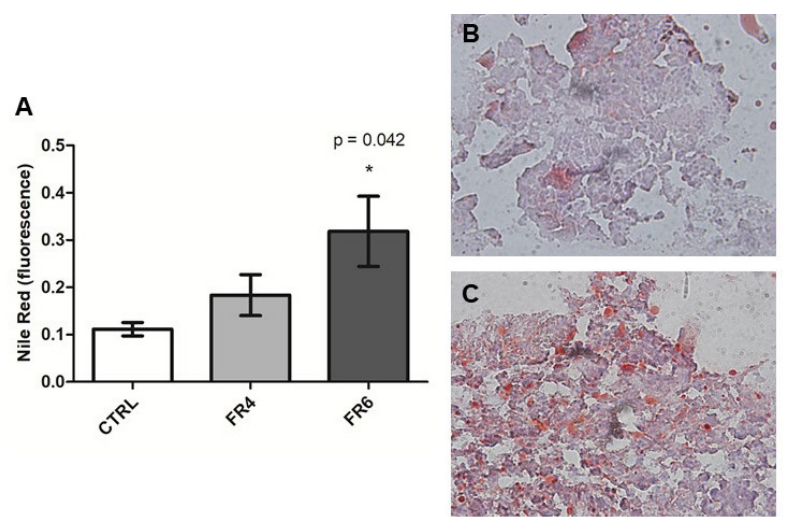

Figure 1: Evaluation of lipid content in daily exposures. A: Quantification of hepatic lipids by Nile Red. The FR6 group presented an increase in lipids concentration when compared to the FR4 and CTRL groups. A statistical analysis was performed by the Kruskal Wallis test. B-C: Staining with Oil Red O. Unlike the CTRL group (B), the FR6 group presents red-colored lipid droplets $(C)$. Magnification of 100x. 


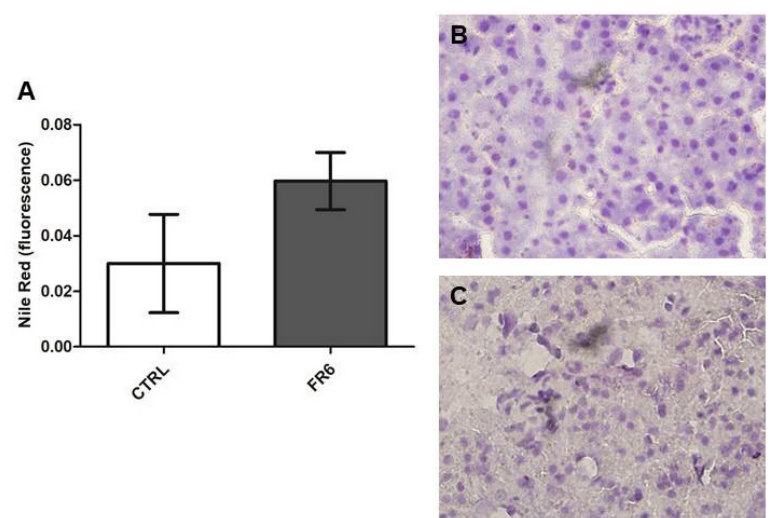

Figure 2: Evaluation of lipid content in exposures every 2 days. A: Quantification of hepatic lipids by Nile Red staining. The FR6 group presented no statistical difference when compared to the CTRL group. A statistical analysis was performed by the Mann Whitney test. B-C: Staining with Oil Red O. The FR6 group (B) did not present difference in lipid deposition compared to the CTRL group (C). Magnification of 100x.

\section{DISCUSSION}

NAFLD is currently the most common chronic liver disease in Western countries, since one billion people are estimated of currently suffering from any form of NAFLD ${ }^{13,14}$. Hepatic steatosis is an important concern in global health, because sedentary lifestyle, modern Western diet, and some pharmacological agents have been identified as critical causes of NAFLD ${ }^{14}$.

Changes in dietary habits in the last decades resulted in the introduction of sweeteners such as fructose and sucrose. The role of fructose in NAFLD has been questioned due to the increased presence of NAFLD in parallel with the rapid consumption of fructose ${ }^{15-17}$. Fructose is taken up into the portal system by hepatocytes via glucose transporter members 2 and 5 and is metabolized in the glycolytic and lipogenic pathway ${ }^{18,19}$.

There are currently several animal models of hepatic steatosis on exposure to fructose. Spruss et al. ${ }^{20}$ have shown that the addition of $30 \%$ fructose in murine drinking water for 8 weeks causes increased levels of triglycerides, hepatic steatosis, and increased body weight $^{20}$. Sapp et al. used the zebrafish model, they analyzed larvae, and observed that a $4 \%$ concentration of fructose lead to hepatic steatosis ${ }^{21}$.

In this study we evaluated the effect of different concentrations of fructose in adult zebrafish, since this is an excellent study model. Our results showed that daily exposure to $6 \%$ fructose could increase hepatic lipid accumulation in adult zebrafish, but induction with $4 \%$ fructose showed no effects on the liver. However, these results differ from those of Sap et al. ${ }^{21}$, which may be due to the fact that they studied zebrafish larvae and not adult fish. Thereby, we chose the $6 \%$ concentration for the following analyzes.

Although daily exposure to fructose is related to hepatic steatosis, non-daily exposure to fructose in adult zebrafish has not yet been evaluated. Thus, we tested exposure every 2 days, since we believe that this model best reflects the consumption of fructose in the diet of patients with hepatic steatosis. Our results showed no difference in the analysis of hepatic steatosis between the groups.

These previous results provide an indication that it is possible to use adult zebrafish as an animal model for the induction of hepatic steatosis by fructose. The use of zebrafish allows the analysis of other parameters such as glycemia, total cholesterol, triglycerides, and high-density protein (HDL), as well as the development of molecular studies. Since the objective of this study was first to determine the amount of fructose ( $4 \%$ or $6 \%$ ) as well as the type of induction (daily or every 2 days) necessary for generation of hepatic steatosis, we hardly performed histological analyzes to use a minimum number of animals. Even so, future studies are necessary to evaluate additional parameters related to animal models of steatosis.

In conclusion, daily exposure to $6 \%$ fructose seems to represent a good experimental model of hepatic steatosis.

\section{REFERENCES}

1. Ouyang X, Cirillo P, Sautin Y, McCall S, Bruchette JL, Diehl AM, et al. Fructose consumption as a risk factor for non-alcoholic fatty liver disease. J Hepatol. 2008;48(6):993-9. http:// dx.doi.org/10.1016/j.jhep.2008.02.011 PMid:18395287.

2. Abdelmalek MF, Suzuki A, Guy C, Unalp-Arida A, Colvin R, Johnson RJ, et al. Increased fructose consumption is associated with fibrosis severity in patients with nonalcoholic fatty liver disease. Hepatology. 2010;51(6):1961-71. http://dx.doi.org/10.1002/hep.23535. PMid:20301112.

3. Lewis JR, Mohanty SR. Nonalcoholic fatty liver disease: a review and update. Dig Dis Sci. 2010;55(3):56078. http://dx.doi.org/10.1007/s10620009-1081-0. PMid:20101463.
4. Than NN, Newsome PN. A concise review of nonalcoholic fatty liver disease. Atherosclerosis. 2015;239(1):192202. http://dx.doi.org/10.1016/j. atherosclerosis.2015.01.001. PMid:25617860.

5. Cotrim HP, Parise ER, Oliveira CPMS, Leite N, Martinelli A, Galizzi J, et al. Nonalcoholic fatty liver disease 
in Brazil. Clinical and histological profile. Ann Hepatol. 2011;10(1):33-7. PMid:21301007.

6. Madero M, Arriaga JC, Jalal D, Rivard C, McFann K, Pérez-Méndez O, et al. The effect of two energy-restricted diets, a low-fructose diet versus a moderate natural fructose diet, on weight loss and metabolic syndrome parameters: a randomized controlled trial. Metabolism. 2011;60(11):15519. http://dx.doi.org/10.1016/j. metabol.2011.04.001. PMid:21621801.

7. Hebbard L, George J. Animal models of nonalcoholic fatty liver disease. Nat Rev Gastroenterol Hepatol. 2011;8(1):35-44. http://dx.doi. org/10.1038/nrgastro.2010.191. PMid:21119613.

8. Takahashi Y, Soejima Y, Fukusato T. Animal models of nonalcoholic fatty liver disease/nonalcoholic steatohepatitis. World J Gastroenterol. 2012;18(19):2300-8. http://dx.doi. org/10.3748/wjg.v18.i19.2300. PMid:22654421.

9. Howe K, Clark MD, Torroja CF, Torrance J, Berthelot C, Muffato $M$, et al. The zebrafish reference genome sequence and its relationship to the human genome. Nature. 2013;496(7446):498-503. http:// dx.doi.org/10.1038/nature12111. PMid:23594743.

10. Goldsmith JR, Jobin C. Think small: zebrafish as a model system of human pathology. J Biomed Biotechnol. 2012;2012:817341. http:// dx.doi.org/10.1155/2012/817341. PMid:22701308.
11. Wilson JM, Bunte RM, Carty AJ. Evaluation of rapid cooling and tricaine methanesulfonate (MS222) as methods of euthanasia in zebrafish (Danio rerio). J Am Assoc Lab Anim Sci. 2009;48(6):785-9. PMid:19930828.

12. Gómez-Lechón MJ, Donato MT, Martínez-Romero A, Jiménez N, Castell JV, O'Connor J-E. A human hepatocellular in vitro model to investigate steatosis. Chem Biol Interact. 2007;165(2):106-16. http:// dx.doi.org/10.1016/j.cbi.2006.11.004 PMid:17188672.

13. Loomba R, Sanyal AJ. The global NAFLD epidemic. Nat Rev Gastroenterol Hepatol. 2013;10(11):686-90. http://dx.doi. org/10.1038/nrgastro.2013.171. PMid:24042449.

14. Willebrords J, Pereira IVA, Maes M, Crespo Yanguas S, Colle I, Van Den Bossche B, et al. Strategies, models and biomarkers in experimental nonalcoholic fatty liver disease research. Prog Lipid Res. 2015;59:10625. http://dx.doi.org/10.1016/j. plipres.2015.05.002. PMid:26073454.

15. Vos MB, Kimmons JE, Gillespie C, Welsh J, Blanck HM. Dietary fructose consumption among US children and adults: the third national health and nutrition examination survey. Medscape J Med. 2008;10(7):160. PMid:18769702.

16. Vos MB, Lavine JE. Dietary fructose in nonalcoholic fatty liver disease. Hepatology. 2013;57(6):2525-31. http://dx.doi.org/10.1002/hep.26299. PMid:23390127.
17. Welsh JA, Karpen S, Vos MB. Increasing prevalence of nonalcoholic fatty liver disease among United States adolescents, 1988-1994 to 2007-2010. J Pediatr. 2013;162(3):496-500.e1. http://dx.doi. org/10.1016/j.jpeds.2012.08.043. PMid:23084707.

18. Lim JS, Mietus-Snyder M, Valente A, Schwarz J-M, Lustig RH. The role of fructose in the pathogenesis of NAFLD and the metabolic syndrome. Nat Rev Gastroenterol Hepatol. 2010;7(5):251-64. http://dx.doi. org/10.1038/nrgastro.2010.41. PMid:20368739.

19. Nomura K, Yamanouchi T. The role of fructose-enriched diets in mechanisms of nonalcoholic fatty liver disease. J Nutr Biochem. 2012;23(3):2038. http://dx.doi.org/10.1016/j. jnutbio.2011.09.006. PMid:22129639.

20. Spruss A, Kanuri G, Wagnerberger S, Haub S, Bischoff SC, Bergheim I. Toll-like receptor 4 is involved in the development of fructoseinduced hepatic steatosis in mice. Hepatology. 2009;50(4):1094-104. http://dx.doi.org/10.1002/hep.23122. PMid:19637282.

21. Sapp V, Gaffney L, EauClaire SF, Matthews RP, EauClaire SF, Matthews RP. Fructose leads to hepatic steatosis in zebrafish that is reversed by mechanistic target of rapamycin (mTOR) inhibition. Hepatology. 2014;60(5):1581-92. http://dx.doi.org/10.1002/hep.27284. PMid:25043405. 